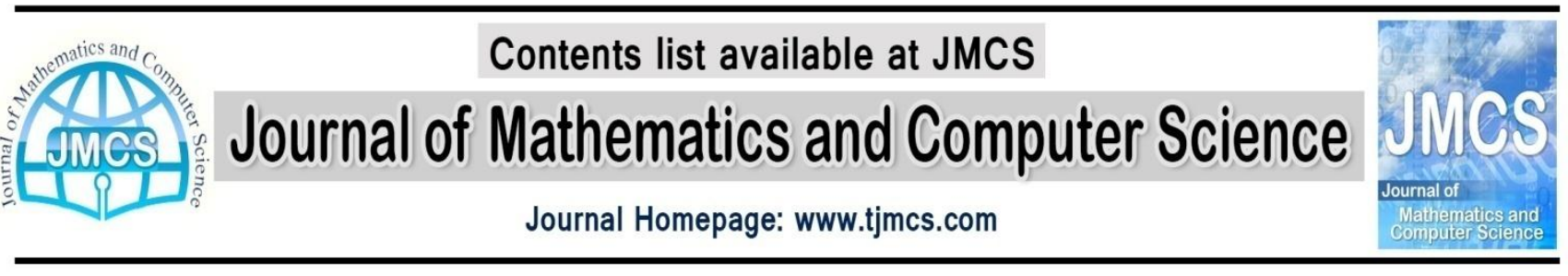

\title{
Efficient Solution of Fractional Initial Value Problems Using Expanding Perturbation Approach
}

\author{
Khosro Sayevand \\ Department of Mathematics, Faculty of Science, Malayer University, Malayer, Iran \\ ksayehvand@malayeru.ac.ir
}

Article history:

Received February 2013

Accepted May 2013

Available online July 2013

\begin{abstract}
In this article, expanding perturbation approach is applied for solving the initial value problems with fractional coordinate derivatives. The fractional derivative is described in the Caputo sense. The response expressions are written in terms of the Mittag-Leffler functions. Convergence of the approach is proved. Comparisons are made to confirm the reliability and effectiveness of the present ideas.
\end{abstract}

Keywords: Expanding perturbation approach, fractional initial value problems, Caputo derivative.

\section{Introduction}

During the last years, numerous papers deal with analytical methods for solving differential equations of fractional order [1-10] appeared in several international journals, e.g. the $\left(G^{\prime} / G\right)$ method [11], the parameter-expanding method [12], Fan Sub-equation Method [13],the Adomian's decomposition method [14,15], the homotopy perturbation technique [16-19]. The homotopy analysis method [20] and the varitional iteration method [21]. In this paper, we recommend a robust analytical method based on expanding perturbation approach $[22,23]$ to solve the fractional initial value problems. We will show that the suggested strategy introduce a powerful improvement for solving this type of problem. 


\subsection{Fractional calculus}

Recently, there has been a great deal of interest in fractional calculus (that is, calculus of integrals and derivatives of any arbitrary real or complex order). Researchers have found many of the physical phenomena can be modeled accurately using the fractional derivatives. For more information about applications of this interesting theory see [1, 2, 3].

Here under some preliminaries and notations regarding fractional calculus presented. For more details see [2].

There are several definitions of a fractional derivative of order $\alpha>0[1,3]$. The two most commonly used definitions are the Riemann-Liouville and Caputo. Each definition uses Riemann-Liouville integration. Riemann-Liouville fractional integration of order $\alpha$ is defined as

$$
\begin{aligned}
& I_{t}^{\alpha} \xi(t)=\frac{1}{\Gamma(\alpha)} \int_{0}^{t} \frac{\xi(\tau)}{(t-\tau)^{1-\alpha}} d \tau, \alpha>0, t>0, \\
& I_{t}^{0} \xi(t)=\xi(t), \\
& I_{x_{k}}^{\alpha_{k}} \xi\left(t_{1}, t_{2}, \ldots, t_{n}\right)=\frac{1}{\Gamma\left(\alpha_{k}\right)} \int_{0}^{t_{k}} \frac{\xi\left(t_{1}, t_{2}, \ldots, t_{k-1}, x_{k}, t_{k+1}, \ldots, t_{n}\right)}{\left(t_{k}-x_{k}\right)^{1-\alpha}} d x_{k},
\end{aligned}
$$

where $\Gamma(\alpha)$ is the well-known Gamma function. The next two equations define RiemannLiouville and Caputo fractional derivatives of order $\alpha$ respectively,

$$
\begin{aligned}
& D_{\mathrm{t}}^{\alpha} \xi(t)=\frac{d^{m}}{d t^{m}} I_{t}^{m-\alpha} \xi(t), m-1<\alpha \leq m, m \in N, \\
& D_{*_{t}}^{\alpha} \xi(t)=\left\{\begin{array}{cr}
I_{t}^{m-\alpha} \xi^{(m)}(t), & m-1<\alpha<m, m \in N, \\
\frac{d^{m}}{d t^{m}} \xi(t), & m=\alpha .
\end{array}\right.
\end{aligned}
$$

In this paper we have chosen to use the Caputo definition, which is a modification of the Riemann - Liouville definition, because when we interpret the fractional derivative in Eq. (1) as a Caputo fractional derivative with suitable conditions on the forcing function $\varepsilon\left(x_{1}, x_{2}, \ldots, x_{n}, t\right)$, the Caputo fractional derivative allows traditional initial and boundary conditions to be included in the formulation of the problem. The reader is advised to consults the geometric and physical interpretation for fractional derivatives and fractional integrals of both the RiemannLiouville and Caputo types in [2]. 


\subsection{Fractional initial value problems}

One very important class of differential equations of fractional order is the fractional initial value problems written in the following form

$$
D^{\alpha} \xi(t)=f(t, \xi(t)), \quad \xi^{(k)}(0)=\xi_{0}^{(k)}, \quad k=0,1,2, \ldots, n-1,
$$

Where $f$ is an arbitrary function, $D^{\alpha}$ denotes the fractional derivative and $\xi_{0}^{(k)}$ are the specified initial conditions.

Theorem 1.2.1. The Eq. (5) is equivalent to the nonlinear Volterra integral equation of the second kind

$$
\xi(t)=\sum_{k=0}^{m-1} \xi_{0}^{(k)} \frac{t^{k}}{k !}+\frac{1}{\Gamma(\alpha)} \int_{0}^{t} \frac{f(x, \xi(x))}{(t-x)^{1-\alpha}} d x .
$$

Proof. See [22; Sayevand and Golbabai, pp. 5]

\subsection{Expanding perturbation approach: Some basic notations}

Definition 1.3.1. The function $f(x, \varepsilon)$ is an approximation to $u(x, \varepsilon)$ uniformly [23, 24] valid to order $\eta(\varepsilon)$ if

$$
\lim _{\varepsilon \rightarrow 0} \frac{u(x, \varepsilon)-f(x, \varepsilon)}{\eta(\varepsilon)}=0 \quad \text { uniformly for } \quad x \in R \text {. }
$$

Definition 1.3.2. Let $f(x)$ and $g(x)$ be two functions defined on the real numbers. We set $f(\varepsilon)=O(g(\varepsilon)) \quad \varepsilon \rightarrow 0$, if

$$
\lim _{\varepsilon \rightarrow 0} \frac{f(\varepsilon)}{g(\varepsilon)}<\infty
$$

Definition 1.3.3. We set $f(\varepsilon)=o(g(\varepsilon)) \quad \varepsilon \rightarrow 0$, if

$$
\lim _{\varepsilon \rightarrow 0} \frac{f(\varepsilon)}{g(\varepsilon)}=0
$$

Now, consider the following function

$$
f(\varepsilon) \sim \sum_{n=0}^{\infty} a_{n} \delta_{n}(\varepsilon), \varepsilon \rightarrow 0
$$

Such that $\delta_{n}(\varepsilon)=o\left(\delta_{n-1}(\varepsilon)\right)$. We say that, $f$ has an asymptotic perturbation expansion. 
Theorem 1.3.1. Under the assumption of the relations (9), (10), we have

$$
a_{n}=\lim _{\varepsilon \rightarrow 0} \frac{f(\varepsilon)-\sum_{m=0}^{n-1} a_{m} \delta_{m}(\varepsilon)}{\delta_{n}(\varepsilon)} .
$$

Proof. From Eq. (10) we obtain

$$
\frac{f(\varepsilon)-a_{0} \delta_{0}(\varepsilon)}{\delta_{1}(\varepsilon)} \sim a_{1}+a_{2} \frac{\delta_{2}(\varepsilon)}{\delta_{1}(\varepsilon)}+\ldots
$$

Whence, (12) implies that

$$
\lim _{\varepsilon \rightarrow 0} \frac{f(\varepsilon)-a_{0} \delta_{0}(\varepsilon)}{\delta_{1}(\varepsilon)}=a_{1}
$$

Continuing, the process, the proof can be completed.

\section{The idea of the suggested scheme}

In this section, we shall illustrate the applicability of our proposed scheme (EPM) to fractional initial value problems.

Consider the following initial value problem

$$
\begin{aligned}
& D^{\alpha} \xi(t)=f(t, \xi(t)), \\
& \xi^{(k)}(0)=\xi_{0}^{(k)}, B\left(\varepsilon, \varepsilon_{t}\right)=0, \quad k=0,1,2, \ldots, n-1,
\end{aligned}
$$

Where $B$ is boundary operator and the derivatives is described in the Caputo sense. Eq. (14) can be written in the following form

$$
f(t, \xi(t))=g(t, \xi(t))-L[f(t, \xi(t))]-N[f(t, \xi(t))],
$$

where, $L$ is a linear part, $N$ is nonlinear part and $g$ is a known analytical function. By the same manipulation as given in Section (1) and Theorem 1.2.1. We obtain:

$$
\begin{aligned}
& \left(\xi_{0}(t)\right)_{t}^{\alpha}=0 \\
& \left(\xi_{1}(t)\right)_{t}^{\alpha}=-L \xi_{0}(t)-S_{0}\left(\xi_{0}(t)\right)+g(t) \\
& \left(\xi_{j-1}(t)\right)_{t}^{\alpha}=-L \xi_{j-2}(t)-S_{j-2}\left(\xi_{0}(t), \ldots, \xi_{j-2}(t)\right), j=2,3, \ldots .
\end{aligned}
$$

Where

$$
N[f(t, \xi(t))]=S\left(\sum_{n=0}^{\infty} a_{n} \xi_{n}(t)\right)=\sum_{n=0}^{\infty} a_{n} S_{n},
$$


and

$$
S_{l}=S_{l}\left(\xi_{0}(t), \xi_{1}(t), \ldots, \xi_{l}(t)\right) .
$$

Consequently, we obtain the following relations:

$\xi_{0}(t)=0$,

$$
\begin{aligned}
& \xi_{1}(t)=-L I_{t}^{\alpha} \xi_{0}(t)-S_{0}\left(\xi_{0}(t)\right)+g(t)+I_{t}^{-n} \sum_{k=0}^{n-1} \xi_{j-1}(0)_{t}^{(k)} \frac{t^{k}}{k !} \\
& \xi_{j-1}(t)=-L I_{t}^{\alpha} \xi_{j-2}(t)-S_{j-2}\left(\xi_{0}(t), \ldots, \xi_{j-2}(t)\right)+I_{t}^{-n} \sum_{k=0}^{n-1} \xi_{j-1}(0)_{t}^{(k)} \frac{t^{k}}{k !}, j=2,3, \ldots
\end{aligned}
$$

\section{Application and numerical results}

To give a clear overview of the suggested scheme, the following examples are presented. All the results are calculated by using the symbolic calculus software Mathematica.

Example 3.1. Consider the following fractional Riccati equation

$$
\xi_{t}^{\alpha}(t)=1-\xi^{2}(t), 0<\alpha \leq 1,
$$

subject to the initial condition

$$
\xi(0)=0 .
$$

The exact solution of Eq. (18) for $\alpha=1$, is

$$
\xi(t)=\frac{e^{2 t}-1}{e^{2 t}+1}
$$

By the same manipulation as given in pervious section we obtain

$$
\xi(t)=\frac{1}{\Gamma(\alpha+1)} t^{\alpha}-\frac{\Gamma(2 \alpha+1)}{\alpha^{2} \Gamma(3 \alpha+1)} t^{3 \alpha}+\frac{16 \Gamma(2 \alpha) \Gamma(4 \alpha)}{\Gamma(3 \alpha+1) \Gamma(5 \alpha+1)} t^{5 \alpha}+\ldots
$$

Now, assume that the leading term has the form

$$
\xi(t)=\sigma(t)\left(\frac{1}{\kappa \varepsilon^{v}}\right), \kappa, \varepsilon, v \in \mathfrak{R} .
$$

Where $\sigma$ is a suitable function. Substituting (24) in to (23) cause that we have some different approximation. For instance, the following expression can be obtained easily when $\alpha=1$,

$$
\xi(t)=2 t-3.009 t^{3}+7.243 t^{5}-19.615 t^{7}+55.963 t^{9}+O\left(t^{11}\right) .
$$


Figure 3.1. Shows that the solution obtained by our scheme (EPM) which are in excellent agreements with exact solution.

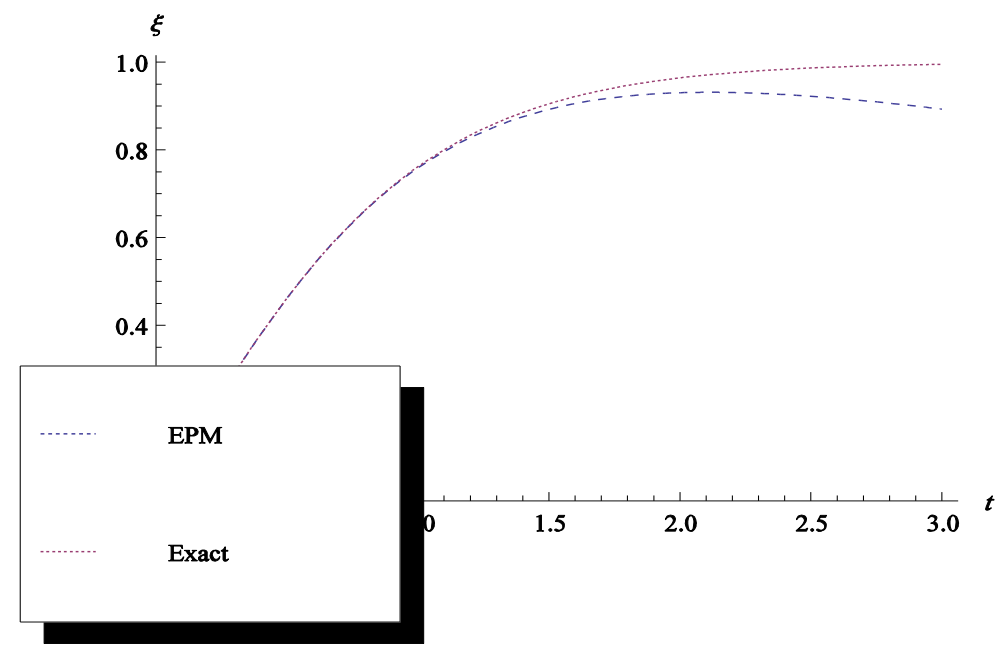

Figure 3.1.

Mittag-Leffler function $E_{\alpha}(z)$ is defined by the following series representation, valid in the whole complex plane [25-28]

$$
E_{\alpha}(z)=\sum_{k=0}^{\infty} \frac{z^{k}}{\Gamma(1+\alpha k)},
$$

Eq. (25) is a convergence series. It is easily verified that our obtained solution in Eq. (22) are convergence and Mittag-Leffler stable.

Example 3.2. Consider the following initial value problem

$$
D_{*_{t}}^{3} \xi(t)+D_{*_{t}}^{2.5} \xi(t)+\xi^{2}(t)=t^{4},
$$

Subject to the initial condition

$$
\xi(t)=t^{2},
$$

Using our approach, we get the $\xi(t)=t^{2}$ as approximate solution, which is in excellent agreement with the analytical solution obtained by Jafari et al. [27].

\section{Concluding remarks}

In this study, the approximate solution of the fractional initial value problems, are obtained using expanding perturbation approach. The fractional derivative are described in the Caputo sense. The solutions are given in the Mittag-Leffler stable series. The obtained results show that our solution is in high agreement with the analytical solution obtained by Adomian decomposition method and homotopy perturbation technique. Finally, our out puts, reveal that this proposed strategy provides highly accurate solutions. 


\section{References}

[1] I. Podlubny, Fractional differential equations, Academic Press, San Diego, 1999.

[2] J. Tenreiro Machado, V. Kiryakova, F. Mainardi, Recent history of fractional calculus, Commun Nonlinear Sci Numer Simu. 16 (2011) 1140-1153.

[3] I. Podlubny, Geometric and physical interpretation of fractional integration and fractional differentiation, Fract. Calculus Appl. Anal. 5 (2002) 367-386.

[4] F.Mainardi, The fundamental solutions for the fractional diffusion-wave equation, Appl.Math. Lett. 9 (1996) 23-28.

[5] F. Mainardi, Fractional calculus: some basic problems in continuum and statistical mechanics, New York, Springer, 1997 p.291-348.

[6] J. H. He, Nonlinear oscillation with fractional derivative and its applications, in: International conference on vibrating engineering'98, (1998), Dalian, China, pp. 288-291.

[7] B. Ghazanfari, A. Sepahvandzadeh, Adomian decomposition method for solving fractional Bratu-type equations, J. Math. Comput. Sci. 8 (3) (2014) 236-244.

[8] M.Fardi, K.Sayevand, Homotopy analysis method: A fresh view on Benjamin-Bona-MahonyBurgers equation, J. Math. Comput. Sci. 4(3) (2012) 494-501.

[9] A. Golbabai, K. Sayevand, Analytical modelling of fractional advection-dispersion equation defined in a bounded space, 53 (2011) 1708-1718.

[10] K. Sayevand, A. Golbabai, A. Yildirim, Analysis of differential equation of fractional order, Appli. Math. Modell. 36 (2012) 4356-4364.

[11] H. Jafari, N. Kadkhoda, E. Salehpoor, Application of $\left(G^{\prime} / G\right)$-expansion method to nonlinear lienard equation, Indian J. Sci. Tech. 5 (4)(2012) 2554-2556.

[12] J. H. He, D. H. Shou, Application of parameter-expanding method to strongly nonlinear oscillators, Int. J. Nonlinear Sci. Numer. Simul. 8 (2007) 121-124.

[13] H. Jafari, M. Ghorbani, C. M. Khalique, Exact Travelling Wave Solutions for Isothermal Magnetostatic Atmospheres by Fan Sub-equation Method, Abst. Appl. Anal. 2012, Article ID 962789, 11 pages doi:10.1155/2012/962789.

[14] G. Adomian, Solving frontier problems of physics: The decomposition method, Kluwer, (1994). 
[15] S.Momani, K. Al-Khald, Numerical solution of the decomposition method, Appl.Math. Comput. 162(2005) 1351-1365.

[16] J. H. He, Homotopy perturbation technique, Comput. Methods Appl. Mech. Eng. 178 (1999) 257-262.

[17] S. Momani, Z. Odibat, Homotopy perturbation method for nonlinear partial differential equations of fractional order, Phys. Lett. A 365 (2007) 345-350.

[18] Z. Odibat, Compact structures in a class of nonlinearly dispersive equations with timefractional derivatives, Appl. Math. Comput. 205 (2008) 273-280.

[19] Y. Khan, N. Faraz, S. Kumar, A. Yildirim, A coupling method of homotopy method and Laplace transform for fractional modells, U.P.B. Sci. Bull., Series A Appl. Math. Phys, 74 (1) (2012) 57 - 68.

[20] H. Jafari, K. Sayevand, H. Tajadidi, D. Baleanu, Homotopy analysis method for solving Abel differential equation of fractional order, Cent. Europ. J. Phys. (2013),Doi: 10.24781.

[21] J. H. He, Variational iteration method-Some recent results and new interpretations, J. Comput. Appl. Math. (207) (1) (2007) 3-17.

[22] K.Sayevand, A.Golbabai, Analysis of differential equations of fractional order, Appli. Mat. Model. 36 (2012) 4356-4364.

[23] A. H. Nayefeh, Introduction to perturbation techniques, John Wiley, 1993.

[24] N. N. Bogoliubov, Y. A. Mitropolsky, Asymptotic methods in the theory of nonlinear silliations, Gordon and Breach, New Work, 1961.

[25] F. Mainardi, R. Gorenflo, On Mittag-Leffler-type functions in fractional evolution processes, J. Comput. Appl. Math. 118 (2000) 283-299.

[26] A. Golbabai, K. Sayevand, The homotopy perturbation method for multi order time fractional differential equations, Nonlinear Sci. Lett. A 2 (2010) 141-147.

[27] V. Daftardar, H. Jafari, Solving a multi-Order fractional differential equations using Adomian decomposition, Appl. Math. Comput. 189(2007) 541-548.

[28] S. Momani, S. Hadid, Lyapunov stability solutions of fractional integro differential equations, Int. J. Math. Mathematical Scie. 47 (2004) 2503-2507. 